\title{
A NOTE ON REPRESENTATIONS OF SOME AFFINE VERTEX ALGEBRAS OF TYPE $D$
}

\author{
OZREN PERŠE \\ University of Zagreb, Croatia
}

\begin{abstract}
In this note we construct a series of singular vectors in universal affine vertex operator algebras associated to $D_{\ell}^{(1)}$ of levels $n-\ell+1$, for $n \in \mathbb{Z}_{>0}$. For $n=1$, we study the representation theory of the quotient vertex operator algebra modulo the ideal generated by that singular vector. In the case $\ell=4$, we show that the adjoint module is the unique irreducible ordinary module for simple vertex operator algebra $L_{D_{4}}(-2,0)$. We also show that the maximal ideal in associated universal affine vertex algebra is generated by three singular vectors.
\end{abstract}

\section{IntRoduCtion}

The classification of irreducible modules for simple vertex operator algebra $L_{\mathfrak{g}}(k, 0)$ associated to affine Lie algebra $\hat{\mathfrak{g}}$ of level $k$ is still an open problem for general $k \in \mathbb{C}\left(k \neq-h^{\vee}\right)$. This problem is connected with the description of the maximal ideal in the universal affine vertex algebra $N_{\mathfrak{g}}(k, 0)$. One approach to this classification problem is through construction of singular vectors in $N_{\mathfrak{g}}(k, 0)$.

The known (non-generic) cases include positive integer levels (cf. [14, $19,20]$ ) and some special cases of rational admissible levels, in the sense of Kac and Wakimoto ([17]) (cf. [1,3,4,6,9,21,22]). It turns out that negative integer levels also have some interesting properties. They appeared in bosonic realizations in [10], and also recently in the context of conformal embeddings (cf. [5]).

In this note we study a vertex operator algebra associated to affine Lie algebra of type $D_{\ell}^{(1)}$ and negative integer level $-\ell+2$. This level appeared in

2010 Mathematics Subject Classification. 17B69, 17B67, 81R10.

Key words and phrases. Vertex operator algebra, affine Kac-Moody algebra, Zhu's algebra. 
[5] in the context of conformal embedding of $L_{B_{\ell-1}}(-\ell+2,0)$ into $L_{D_{\ell}}(-\ell+$ $2,0)$. This conformal embedding is in some sense similar to the conformal embedding of $L_{D_{\ell}}\left(-\ell+\frac{3}{2}, 0\right)$ into $L_{B_{\ell}}\left(-\ell+\frac{3}{2}, 0\right)$.

We will show that there are also similarities in singular vectors in universal affine vertex algebras $N_{B_{\ell}}\left(-\ell+\frac{3}{2}, 0\right)$ (studied in [21]) and $N_{D_{\ell}}(-\ell+2,0)$. More generally, we construct a series of singular vectors

$$
v_{n}=\left(\sum_{i=2}^{\ell} e_{\epsilon_{1}-\epsilon_{i}}(-1) e_{\epsilon_{1}+\epsilon_{i}}(-1)\right)^{n} \mathbf{1}
$$

in $N_{D_{\ell}}(n-\ell+1,0)$, for any $n \in \mathbb{Z}_{>0}$. For $n=1$, we study the representation theory of the quotient $N_{D_{\ell}}(-\ell+2,0)$ modulo the ideal generated by $v_{1}$. Using the methods from $[1,2,4,20]$, we obtain the classification of irreducible weak modules in the category $\mathcal{O}$ for that vertex algebra. It turns out that there are infinitely many of these modules.

In the special case $\ell=4$, we obtain the classification of irreducible weak modules from the category $\mathcal{O}$ for simple vertex operator algebra $L_{D_{4}}(-2,0)$. This vertex algebra also appeared in [5] in the context of conformal embedding of $L_{G_{2}}(-2,0)$ into $L_{D_{4}}(-2,0)$. It follows that there are finitely many irreducible weak $L_{D_{4}}(-2,0)$-modules from the category $\mathcal{O}$, that the adjoint module is the unique irreducible ordinary $L_{D_{4}}(-2,0)$-module, and that every ordinary $L_{D_{4}}(-2,0)$-module is completely reducible. We also show that the maximal ideal in $N_{D_{4}}(-2,0)$ is generated by three singular vectors.

\section{Preliminaries}

We assume that the reader is familiar with the notion of vertex operator algebra (cf. [7, 11-14,16,18,19]) and Kac-Moody algebra (cf. [15]).

Let $V$ be a vertex operator algebra. Denote by $A(V)$ the associative algebra introduced in [23], called the Zhu's algebra of $V$. As a vector space, $A(V)$ is a quotient of $V$, and we denote by $[a]$ the image of $a \in V$ under the projection of $V$ onto $A(V)$. We recall the following fundamental result from $[23]$ :

Proposition 2.1. The equivalence classes of the irreducible $A(V)$ modules and the equivalence classes of the irreducible $\mathbb{Z}_{+}$-graded weak $V$ modules are in one-to-one correspondence.

Let $\mathfrak{g}$ be a simple Lie algebra with a triangular decomposition $\mathfrak{g}=\mathfrak{n}_{-} \oplus$ $\mathfrak{h} \oplus \mathfrak{n}_{+}$, and $\hat{\mathfrak{g}}$ the (untwisted) affine Lie algebra associated to $\mathfrak{g}$. Denote by $V(\mu)$ the irreducible highest weight $\mathfrak{g}$-module with highest weight $\mu$, and by $L(k, \mu)$ the irreducible highest weight $\hat{\mathfrak{g}}$-module with highest weight $k \Lambda_{0}+\mu$.

Furthermore, denote by $N(k, 0)$ (or $\left.N_{\mathfrak{g}}(k, 0)\right)$ the universal affine vertex algebra associated to $\hat{\mathfrak{g}}$ of level $k \in \mathbb{C}$. For $k \neq-h^{\vee}, N(k, 0)$ is a vertex operator algebra with Segal-Sugawara conformal vector, and $L(k, 0)$ is a 
simple vertex operator algebra. The Zhu's algebra of $N(k, 0)$ was determined in [14]:

Proposition 2.2. The associative algebra $A(N(k, 0))$ is canonically isomorphic to $U(\mathfrak{g})$. The isomorphism is given by $F: A(N(k, 0)) \rightarrow U(\mathfrak{g})$

$$
F\left(\left[x_{1}\left(-n_{1}-1\right) \cdots x_{m}\left(-n_{m}-1\right) \mathbf{1}\right]\right)=(-1)^{n_{1}+\cdots+n_{m}} x_{m} \cdots x_{1},
$$

for any $x_{1}, \ldots, x_{m} \in \mathfrak{g}$ and any $n_{1}, \ldots, n_{m} \in \mathbb{Z}_{+}$.

We have:

Proposition 2.3. Assume that a $\hat{\mathfrak{g}}$-submodule $J$ of $N(k, 0)$ is generated by $m$ singular vectors $\left(m \in \mathbb{Z}_{>0}\right)$, i.e. $J=U(\hat{\mathfrak{g}}) v^{(1)}+\ldots+U(\hat{\mathfrak{g}}) v^{(m)}$. Then

$$
A(N(k, 0) / J) \cong U(\mathfrak{g}) / I
$$

where $I$ is the two-sided ideal of $U(\mathfrak{g})$ generated by $u^{(1)}=F\left(\left[v^{(1)}\right]\right), \ldots, u^{(m)}=$ $F\left(\left[v^{(m)}\right]\right)$.

Let $J=U(\hat{\mathfrak{g}}) v^{(1)}+\ldots+U(\hat{\mathfrak{g}}) v^{(m)}$ be a $\hat{\mathfrak{g}}$-submodule of $N(k, 0)$ generated by singular vectors $v^{(1)}, \ldots, v^{(m)}$. Now we recall the method from $[1,2,4,20]$ for the classification of irreducible $A(N(k, 0) / J)$-modules from the category $\mathcal{O}$ by solving certain systems of polynomial equations.

Denote by ${ }_{L}$ the adjoint action of $U(\mathfrak{g})$ on $U(\mathfrak{g})$ defined by $X_{L} f=[X, f]$ for $X \in \mathfrak{g}$ and $f \in U(\mathfrak{g})$. Let $R^{(j)}$ be a $U(\mathfrak{g})$-submodule of $U(\mathfrak{g})$ generated by the vector $u^{(j)}=F\left(\left[v^{(j)}\right]\right)$ under the adjoint action, for $j=1, \ldots, m$. Clearly, $R^{(j)}$ is an irreducible highest weight $U(\mathfrak{g})$-module. Let $R_{0}^{(j)}$ be the zero-weight subspace of $R^{(j)}$.

The next proposition follows from $[1,4,20]$ :

Proposition 2.4. Let $V(\mu)$ be an irreducible highest weight $U(\mathfrak{g})$-module with the highest weight vector $v_{\mu}$, for $\mu \in \mathfrak{h}^{*}$. The following statements are equivalent:

(1) $V(\mu)$ is an $A(N(k, 0) / J)$-module,

(2) $R^{(j)} V(\mu)=0$, for every $j=1, \ldots, m$,

(3) $R_{0}^{(j)} v_{\mu}=0$, for every $j=1, \ldots, m$. that

Let $r \in R_{0}^{(j)}$. Clearly there exists the unique polynomial $p_{r} \in S(\mathfrak{h})$ such

$$
r v_{\mu}=p_{r}(\mu) v_{\mu} .
$$

Set $\mathcal{P}_{0}^{(j)}=\left\{p_{r} \mid r \in R_{0}^{(j)}\right\}$, for $j=1, \ldots, m$. We have:

COROLlary 2.5. There is one-to-one correspondence between

(1) irreducible $A(N(k, 0) / J)$-modules from the category $\mathcal{O}$,

(2) weights $\mu \in \mathfrak{h}^{*}$ such that $p(\mu)=0$ for all $p \in \mathcal{P}_{0}^{(j)}$, for every $j=$ $1, \ldots, m$. 
In the case $m=1$, we use the notation $R, R_{0}$ and $\mathcal{P}_{0}$ for $R^{(1)}, R_{0}^{(1)}$ and $\mathcal{P}_{0}^{(1)}$, respectively.

\section{Vertex operator Algebra Associated to $D_{\ell}^{(1)}$ OF LEVEL $-\ell+2$}

In this section we study the representation theory of the quotient of universal affine vertex operator algebra associated to $D_{\ell}^{(1)}$ of level $-\ell+2$, modulo the ideal generated by a singular vector of conformal weight two.

Denote by $\mathfrak{g}$ the simple Lie algebra of type $D_{\ell}$. We fix the root vectors for $\mathfrak{g}$ as in $[8,10]$. We have:

THEOREM 3.1. Vector

$$
v_{n}=\left(\sum_{i=2}^{\ell} e_{\epsilon_{1}-\epsilon_{i}}(-1) e_{\epsilon_{1}+\epsilon_{i}}(-1)\right)^{n} \mathbf{1}
$$

is a singular vector in $N_{D_{\ell}}(n-\ell+1,0)$, for any $n \in \mathbb{Z}_{>0}$.

ProOF. Direct verification of relations $e_{\epsilon_{k}-\epsilon_{k+1}}(0) v_{n}=0$, for $k=$ $1, \ldots, \ell-1, e_{\epsilon_{\ell-1}+\epsilon_{\ell}}(0) v_{n}=0$ and $f_{\epsilon_{1}+\epsilon_{2}}(1) v_{n}=0$.

In the case $n=1$, we obtain the singular vector

$$
v=\sum_{i=2}^{\ell} e_{\epsilon_{1}-\epsilon_{i}}(-1) e_{\epsilon_{1}+\epsilon_{i}}(-1) \mathbf{1}
$$

in $N_{D_{\ell}}(-\ell+2,0)$.

REMARK 3.2. Vector $v$ from relation (3.1) has a similar formula as singular vector

$$
-\frac{1}{4} e_{\epsilon_{1}}(-1)^{2} \mathbf{1}+\sum_{i=2}^{\ell} e_{\epsilon_{1}-\epsilon_{i}}(-1) e_{\epsilon_{1}+\epsilon_{i}}(-1) \mathbf{1}
$$

for $B_{\ell}^{(1)}$ in $N_{B_{\ell}}\left(-\ell+\frac{3}{2}, 0\right)$. The representation theory of the quotient of $N_{B_{\ell}}\left(-\ell+\frac{3}{2}, 0\right)$ modulo the ideal generated by that vector was studied in [21].

We will consider representations of the vertex operator algebra

$$
\mathcal{V}_{D_{\ell}}(-\ell+2,0)=\frac{N_{D_{\ell}}(-\ell+2,0)}{U(\hat{\mathfrak{g}}) v}
$$

Proposition 2.3 gives:

Proposition 3.3. The associative algebra $A\left(\mathcal{V}_{D_{\ell}}(-\ell+2,0)\right)$ is isomorphic to the algebra $U(\mathfrak{g}) / I$, where $I$ is the two-sided ideal of $U(\mathfrak{g})$ generated by

$$
u=\sum_{i=2}^{\ell} e_{\epsilon_{1}-\epsilon_{i}} e_{\epsilon_{1}+\epsilon_{i}} \text {. }
$$


We have the following classification:

TheOREM 3.4. For any subset $S=\left\{i_{1}, \ldots, i_{k}\right\} \subseteq\{1,2, \ldots, \ell-2\}$, $i_{1}<\cdots<i_{k}$, and $t \in \mathbb{C}$, we define weights

$$
\begin{aligned}
& \mu_{S, t}=\sum_{j=1}^{k}\left(i_{j}+2 \sum_{s=j+1}^{k}(-1)^{s-j} i_{s}+(-1)^{k-j+1}(t+\ell-1)\right) \omega_{i_{j}}+t \omega_{\ell-1}, \\
& \mu_{S, t}^{\prime}=\sum_{j=1}^{k}\left(i_{j}+2 \sum_{s=j+1}^{k}(-1)^{s-j} i_{s}+(-1)^{k-j+1}(t+\ell-1)\right) \omega_{i_{j}}+t \omega_{\ell},
\end{aligned}
$$

where $\omega_{1}, \ldots, \omega_{\ell}$ are fundamental weights for $\mathfrak{g}$. Then the set

$$
\left\{L_{D_{\ell}}\left(-\ell+2, \mu_{S, t}\right), L_{D_{\ell}}\left(-\ell+2, \mu_{S, t}^{\prime}\right) \mid S \subseteq\{1,2, \ldots, \ell-2\}, t \in \mathbb{C}\right\}
$$

provides the complete list of irreducible weak $\mathcal{V}_{D_{\ell}}(-\ell+2,0)$-modules from the category $\mathcal{O}$.

Proof. We use the method for classification of irreducible $A\left(\mathcal{V}_{D_{\ell}}(-\ell+\right.$ $2,0)$ )-modules in the category $\mathcal{O}$ from Corollary 2.5. In this case $R \cong$ $V_{D_{\ell}}\left(2 \omega_{1}\right)$, and similarly as in [21, Lemma 28] one obtains that

$$
\operatorname{dim} R_{0}=\ell-1 \text {. }
$$

Furthermore, one obtains by direct calculation that

$$
\begin{aligned}
& \left(f_{\epsilon_{1}-\epsilon_{2}} f_{\epsilon_{1}+\epsilon_{2}}\right)_{L} u \in p_{1}(h)+U(\mathfrak{g}) \mathfrak{n}_{+}, \\
& \left(f_{\epsilon_{1}-\epsilon_{i+1}} f_{\epsilon_{1}+\epsilon_{i+1}}-f_{\epsilon_{1}-\epsilon_{i}} f_{\epsilon_{1}+\epsilon_{i}}\right)_{L} u \in p_{i}(h)+U(\mathfrak{g}) \mathfrak{n}_{+}, i=2, \ldots, \ell-1,
\end{aligned}
$$

where

$$
p_{i}(h)=h_{i}\left(h_{\epsilon_{i}+\epsilon_{i+1}}+\ell-i-1\right), \quad \text { for } i=1, \ldots, \ell-1
$$

are linearly independent polynomials in $\mathcal{P}_{0}$. Here $h_{i}(i=1, \ldots, \ell)$ denote the simple coroots for $\mathfrak{g}$ and

$$
h_{\epsilon_{i}+\epsilon_{i+1}}=h_{i}+2 h_{i+1}+\ldots+2 h_{\ell-2}+h_{\ell-1}+h_{\ell}, \quad \text { for } i<\ell-1 .
$$

Corollary 2.5 now implies that the highest weights of irreducible $A\left(\mathcal{V}_{D_{\ell}}(-\ell+\right.$ $2,0)$ )-modules from the category $\mathcal{O}$ are given as solutions of polynomial equations

$$
p_{i}(h)=0, i=1, \ldots, \ell-1 .
$$

First we note that for $i=\ell-1$, we obtain the equation

$$
h_{\ell-1} h_{\ell}=0 \text {. }
$$

Thus, either $h_{\ell-1}=0$ or $h_{\ell}=0$. Assume first that $h_{\ell-1}=0$, and let $S=\left\{i_{1}, \ldots, i_{k}\right\}, i_{1}<\ldots<i_{k}$ be the subset of $\{1,2, \ldots, \ell-2\}$ such that 
$h_{i}=0$ for $i \notin S$ and $h_{i} \neq 0$ for $i \in S$. Then we have the system

$$
\begin{aligned}
& h_{i_{1}}+2 h_{i_{2}}+\ldots+2 h_{i_{k}}+h_{\ell}+\ell-i_{1}-1=0, \\
& h_{i_{2}}+2 h_{i_{3}}+\ldots+2 h_{i_{k}}+h_{\ell}+\ell-i_{2}-1=0,
\end{aligned}
$$

$$
\begin{aligned}
& h_{i_{k-1}}+2 h_{i_{k}}+h_{\ell}+\ell-i_{k-1}-1=0, \\
& h_{i_{k}}+h_{\ell}+\ell-i_{k}-1=0 .
\end{aligned}
$$

The solution of this system is given by

$$
\begin{aligned}
& h_{i_{j}}=i_{j}+2 \sum_{s=j+1}^{k}(-1)^{s-j} i_{s}+(-1)^{k-j+1}(t+\ell-1), \text { for } j=1, \ldots, k ; \\
& h_{\ell}=t \quad(t \in \mathbb{C}) .
\end{aligned}
$$

It follows that $V_{D_{\ell}}\left(\mu_{S, t}^{\prime}\right)$ is an irreducible $A\left(\mathcal{V}_{D_{\ell}}(-\ell+2,0)\right)$-module. Similarly, the case $h_{\ell}=0$ gives that $V_{D_{\ell}}\left(\mu_{S, t}\right)$ is irreducible $A\left(\mathcal{V}_{D_{\ell}}(-\ell+2,0)\right)$-module. We conclude that the set

$$
\left\{V_{D_{\ell}}\left(\mu_{S, t}\right), V_{D_{\ell}}\left(\mu_{S, t}^{\prime}\right) \mid S \subseteq\{1,2, \ldots, \ell-2\}, t \in \mathbb{C}\right\}
$$

provides the complete list of irreducible $A\left(\mathcal{V}_{D_{\ell}}(-\ell+2,0)\right)$-modules from the category $\mathcal{O}$. The claim of theorem now follows from Zhu's theory.

EXAmPLe 3.5. For $\ell=4$, we have subsets $S=\emptyset,\{1\},\{2\},\{1,2\}$ of the set $\{1,2\}$, so we obtain that the set

$$
\begin{aligned}
& \left\{L_{D_{\ell}}\left(-\ell+2, t \omega_{3}\right), L_{D_{\ell}}\left(-\ell+2, t \omega_{4}\right), L_{D_{\ell}}\left(-\ell+2,(-2-t) \omega_{1}+t \omega_{3}\right),\right. \\
& L_{D_{\ell}}\left(-\ell+2,(-2-t) \omega_{1}+t \omega_{4}\right), L_{D_{\ell}}\left(-\ell+2,(-1-t) \omega_{2}+t \omega_{3}\right), \\
& L_{D_{\ell}}\left(-\ell+2,(-1-t) \omega_{2}+t \omega_{4}\right), L_{D_{\ell}}\left(-\ell+2, t \omega_{1}+(-1-t) \omega_{2}+t \omega_{3}\right), \\
& \left.L_{D_{\ell}}\left(-\ell+2, t \omega_{1}+(-1-t) \omega_{2}+t \omega_{4}\right) \mid t \in \mathbb{C}\right\}
\end{aligned}
$$

provides the complete list of irreducible weak $\mathcal{V}_{D_{4}}(-2,0)$-modules from the category $\mathcal{O}$.

Recall that a module for vertex operator algebra is called ordinary if $L(0)$ acts semisimply with finite-dimensional weight spaces. We have:

Corollary 3.6. The set

$$
\left\{L_{D_{\ell}}\left(-\ell+2, t \omega_{\ell-1}\right), L_{D_{\ell}}\left(-\ell+2, t \omega_{\ell}\right) \mid t \in \mathbb{Z}_{\geq 0}\right\}
$$

provides the complete list of irreducible ordinary $\mathcal{V}_{D_{\ell}}(-\ell+2,0)$-modules.

Proof. If $L_{D_{\ell}}(-\ell+2, \mu)$ is an ordinary $\mathcal{V}_{D_{\ell}}(-\ell+2,0)$-module, then $\mu$ is a dominant integral weight. Then $\mu\left(h_{\epsilon_{i}+\epsilon_{i+1}}\right) \in \mathbb{Z}_{\geq 0}$, for $i=1, \ldots, \ell-1$. Relations (3.2) and (3.3) then give that

$$
\mu\left(h_{i}\right)=0, \quad \text { for } i=1, \ldots, \ell-2,
$$


and $\mu\left(h_{\ell-1}\right)=0$ or $\mu\left(h_{\ell}\right)=0$. Thus, $\mu=t \omega_{\ell-1}$ or $\mu=t \omega_{\ell}$, and $t \in \mathbb{Z}_{\geq 0}$ since $\mu$ is a dominant integral weight.

It follows that:

Corollary 3.7. The set of irreducible ordinary $L_{D_{\ell}}(-\ell+2,0)$-modules is a subset of the set

$$
\left\{L_{D_{\ell}}\left(-\ell+2, t \omega_{\ell-1}\right), L_{D_{\ell}}\left(-\ell+2, t \omega_{\ell}\right) \mid t \in \mathbb{Z}_{\geq 0}\right\} .
$$

\section{CASE $\ell=4$}

In this section we study the case $\ell=4$. We determine the classification of irreducible weak $L_{D_{4}}(-2,0)$-modules from the category $\mathcal{O}$. It turns out that there are finitely many of these modules and that the adjoint module is the unique irreducible ordinary $L_{D_{4}}(-2,0)$-module. We also show that the maximal ideal in $N_{D_{4}}(-2,0)$ is generated by three singular vectors.

Denote by $\theta$ the automorphism of $N_{D_{4}}(-2,0)$ induced by the automorphism of the Dynkin diagram of $D_{4}$ of order three such that

$$
\begin{array}{ll}
\theta\left(\epsilon_{1}-\epsilon_{2}\right)=\epsilon_{3}-\epsilon_{4}, & \theta\left(\epsilon_{2}-\epsilon_{3}\right)=\epsilon_{2}-\epsilon_{3}, \\
\theta\left(\epsilon_{3}-\epsilon_{4}\right)=\epsilon_{3}+\epsilon_{4}, & \theta\left(\epsilon_{3}+\epsilon_{4}\right)=\epsilon_{1}-\epsilon_{2} .
\end{array}
$$

Relation (3.1) implies that

$v=\left(e_{\epsilon_{1}-\epsilon_{2}}(-1) e_{\epsilon_{1}+\epsilon_{2}}(-1)+e_{\epsilon_{1}-\epsilon_{3}}(-1) e_{\epsilon_{1}+\epsilon_{3}}(-1)+e_{\epsilon_{1}-\epsilon_{4}}(-1) e_{\epsilon_{1}+\epsilon_{4}}(-1)\right) \mathbf{1}$

is a singular vector in $N_{D_{4}}(-2,0)$. Furthermore,

$$
\begin{aligned}
\theta(v)= & \left(e_{\epsilon_{3}-\epsilon_{4}}(-1) e_{\epsilon_{1}+\epsilon_{2}}(-1)-e_{\epsilon_{2}-\epsilon_{4}}(-1) e_{\epsilon_{1}+\epsilon_{3}}(-1)\right. \\
& \left.+e_{\epsilon_{2}+\epsilon_{3}}(-1) e_{\epsilon_{1}-\epsilon_{4}}(-1)\right) \mathbf{1}
\end{aligned}
$$

and

$$
\begin{aligned}
\theta^{2}(v)= & \left(e_{\epsilon_{3}+\epsilon_{4}}(-1) e_{\epsilon_{1}+\epsilon_{2}}(-1)-e_{\epsilon_{2}+\epsilon_{4}}(-1) e_{\epsilon_{1}+\epsilon_{3}}(-1)\right. \\
& \left.+e_{\epsilon_{1}+\epsilon_{4}}(-1) e_{\epsilon_{2}+\epsilon_{3}}(-1)\right) \mathbf{1}
\end{aligned}
$$

are also singular vectors in $N_{D_{4}}(-2,0)$. We consider the vertex operator algebra

$$
\widetilde{L}_{D_{4}}(-2,0)=\frac{N_{D_{4}}(-2,0)}{J},
$$

where $J$ is the ideal in $N_{D_{4}}(-2,0)$ generated by vectors $v, \theta(v)$ and $\theta^{2}(v)$.

Proposition 2.3 gives that the associative algebra $A\left(\widetilde{L}_{D_{4}}(-2,0)\right)$ is isomorphic to the algebra $U(\mathfrak{g}) / I$, where $I$ is the two-sided ideal of $U(\mathfrak{g})$ generated by $u, \theta(u)$ and $\theta^{2}(u)$, and

$$
u=e_{\epsilon_{1}-\epsilon_{2}} e_{\epsilon_{1}+\epsilon_{2}}+e_{\epsilon_{1}-\epsilon_{3}} e_{\epsilon_{1}+\epsilon_{3}}+e_{\epsilon_{1}-\epsilon_{4}} e_{\epsilon_{1}+\epsilon_{4}} .
$$

Proposition 4.1. We have: 
(i) The set

$\left\{L_{D_{4}}(-2,0), L_{D_{4}}\left(-2,-2 \omega_{1}\right), L_{D_{4}}\left(-2,-2 \omega_{3}\right), L_{D_{4}}\left(-2,-2 \omega_{4}\right), L_{D_{4}}\left(-2,-\omega_{2}\right)\right\}$ provides a complete list of irreducible weak $\widetilde{L}_{D_{4}}(-2,0)$-modules from the category $\mathcal{O}$.

(ii) $L_{D_{4}}(-2,0)$ is the unique irreducible ordinary module for $\widetilde{L}_{D_{4}}(-2,0)$.

Proof. (i) We use the method for classification from Corollary 2.5. In this case $R^{(1)} \cong V_{D_{4}}\left(2 \omega_{1}\right), R^{(2)} \cong V_{D_{4}}\left(2 \omega_{3}\right), R^{(3)} \cong V_{D_{4}}\left(2 \omega_{4}\right)$ and

$$
\operatorname{dim} R_{0}^{(1)}=\operatorname{dim} R_{0}^{(2)}=\operatorname{dim} R_{0}^{(3)}=3 .
$$

Using polynomials from relation (3.2) and automorphisms $\theta$ and $\theta^{2}$, one obtains that the highest weights $\mu$ of $A\left(\widetilde{L}_{D_{4}}(-2,0)\right)$-modules $V_{D_{4}}(\mu)$ are obtained as solutions of these 9 polynomial equations:

$$
\begin{aligned}
& h_{\epsilon_{1}-\epsilon_{2}}\left(h_{\epsilon_{1}+\epsilon_{2}}+2\right)=0, \\
& h_{\epsilon_{2}-\epsilon_{3}}\left(h_{\epsilon_{2}+\epsilon_{3}}+1\right)=0, \\
& h_{\epsilon_{3}-\epsilon_{4}} h_{\epsilon_{3}+\epsilon_{4}}=0, \\
& h_{\epsilon_{3}-\epsilon_{4}}\left(h_{\epsilon_{1}+\epsilon_{2}}+2\right)=0, \\
& h_{\epsilon_{2}-\epsilon_{3}}\left(h_{\epsilon_{1}+\epsilon_{4}}+1\right)=0, \\
& h_{\epsilon_{3}+\epsilon_{4}} h_{\epsilon_{1}-\epsilon_{2}}=0, \\
& h_{\epsilon_{3}+\epsilon_{4}}\left(h_{\epsilon_{1}+\epsilon_{2}}+2\right)=0, \\
& h_{\epsilon_{2}-\epsilon_{3}}\left(h_{\epsilon_{1}-\epsilon_{4}}+1\right)=0, \\
& h_{\epsilon_{1}-\epsilon_{2}} h_{\epsilon_{3}-\epsilon_{4}}=0 .
\end{aligned}
$$

This easily gives that $\mu=0,-2 \omega_{1},-2 \omega_{3},-2 \omega_{4}$ or $-\omega_{2}$, and the claim follows from Zhu's theory.

Claim (ii) follows from the fact that $\mu=0$ is the only dominant integral weight such that $L_{D_{4}}(-2, \mu)$ is in the set given in the claim (i).

We have:

THEOREM 4.2. Vertex operator algebra $\widetilde{L}_{D_{4}}(-2,0)$ is simple, i.e.,

$$
L_{D_{4}}(-2,0)=\frac{N_{D_{4}}(-2,0)}{U(\hat{\mathfrak{g}}) \cdot v+U(\hat{\mathfrak{g}}) \cdot \theta(v)+U(\hat{\mathfrak{g}}) \cdot \theta^{2}(v)} .
$$

Proof. Let $w$ be a singular vector for $\hat{\mathfrak{g}}$ in $\widetilde{L}_{D_{4}}(-2,0)$. The classification result from Proposition 4.1 (ii) implies that $U(\hat{\mathfrak{g}}) . w$ is a highest weight $\hat{\mathfrak{g}}-$ module with highest weight $-2 \Lambda_{0}$ and that $w$ is proportional to $\mathbf{1}$. The claim follows.

We conclude: 
THEOREM 4.3. (i) The set

$\left\{L_{D_{4}}(-2,0), L_{D_{4}}\left(-2,-2 \omega_{1}\right), L_{D_{4}}\left(-2,-2 \omega_{3}\right), L_{D_{4}}\left(-2,-2 \omega_{4}\right), L_{D_{4}}\left(-2,-\omega_{2}\right)\right\}$ provides a complete list of irreducible weak $L_{D_{4}}(-2,0)-$ modules from the category $\mathcal{O}$.

(ii) $L_{D_{4}}(-2,0)$ is the unique irreducible ordinary module for $L_{D_{4}}(-2,0)$.

(iii) Every ordinary $L_{D_{4}}(-2,0)$-module is completely reducible.

Proof. Proposition 4.1 and Theorem 4.2 imply claims (i) and (ii).

(iii) Let $M$ be an ordinary $L_{D_{4}}(-2,0)$-module, and let $w$ be a singular vector for $\hat{\mathfrak{g}}$ in $M$. The classification result from (ii) implies that $U(\hat{\mathfrak{g}}) . w$ is a highest weight $\hat{\mathfrak{g}}$-module with highest weight $-2 \Lambda_{0}$. Claim (ii) also implies that any singular vector in $U(\hat{\mathfrak{g}}) \cdot w$ has highest weight $-2 \Lambda_{0}$ and it is proportional to $w$. Thus, $U(\hat{\mathfrak{g}}) . w$ is an irreducible $\hat{\mathfrak{g}}-$ module and the claim follows.

ACKNOWLEDGEMENTS.

The author thanks Dražen Adamović for his helpful advice and valuable suggestions.

\section{REFERENCES}

[1] D. Adamović, Some rational vertex algebras, Glas. Mat. Ser. III 29(49) (1994), 2540.

[2] D. Adamović, A construction of some ideals in affine vertex algebras, Int. J. Math. Math. Sci. 2003 (2003), 971-980.

[3] D. Adamović, A construction of admissible $A_{1}^{(1)}$-modules of level $-\frac{4}{3}$, J. Pure Appl. Algebra 196 (2005), 119-134.

[4] D. Adamović and A. Milas, Vertex operator algebras associated to modular invariant representations for $A_{1}^{(1)}$, Math. Res. Lett. 2 (1995), 563-575.

[5] D. Adamović and O. Perše, Some general results on conformal embeddings of affine vertex operator algebras, Algebr. Represent. Theory 16 (2013), 51-64.

[6] J. D. Axtell and K.-H. Lee, Vertex operator algebras associated to type G affine Lie algebras, J. Algebra 337 (2011), 195-223.

[7] R. E. Borcherds, Vertex algebras, Kac-Moody algebras, and the Monster, Proc. Nat. Acad. Sci. U.S.A. 83 (1986), 3068-3071.

[8] N. Bourbaki, Iliments de mathimatique. (French) Fasc. XXXVIII: Groupes et alg.bres de Lie. Chapitre VII: Sous-alg.bres de Cartan, iliments riguliers. Chapitre VIII: Alg.bres de Lie semi-simples diployies. Actualitis Scientifiques et Industrielles, No. 1364. Hermann, Paris, 1975.

[9] C. Dong, H. Li and G. Mason, Vertex operator algebras associated to admissible representations of $\widehat{\mathrm{sl}}_{2}$, Comm. Math. Phys. 184 (1997), 65-93.

[10] A. J. Feingold and I. B. Frenkel, Classical affine algebras, Adv. in Math. 56 (1985), $117-172$.

[11] E. Frenkel and D. Ben-Zvi, Vertex algebras and algebraic curves, American Mathematical Society, Providence, 2001.

[12] I. Frenkel, Y.-Z. Huang and J. Lepowsky, On axiomatic approaches to vertex operator algebras and modules, Mem. Amer. Math. Soc. 104, 1993. 
[13] I. Frenkel, J. Lepowsky and A. Meurman, Vertex operator algebras and the Monster, Academic Press, Boston, 1988.

[14] I. Frenkel and Y.-C. Zhu, Vertex operator algebras associated to representations of affine and Virasoro algebras, Duke Math. J. 66 (1992), 123-168.

[15] V. G. Kac, Infinite dimensional Lie algebras, 3rd ed., Cambridge Univ. Press, Cambridge, 1990.

[16] V. G. Kac, Vertex algebras for beginners, AMS, Providence, 1998.

[17] V. Kac and M. Wakimoto, Modular invariant representations of infinite dimensional Lie algebras and superalgebras, Proc. Nat. Acad. Sci. U.S.A. 85 (1988), 4956-4960.

[18] J. Lepowsky and H. Li, Introduction to vertex operator algebras and their representations, Birkhäuser, Boston, 2004.

[19] H.-S. Li, Local systems of vertex operators, vertex superalgebras and modules, J. Pure Appl. Algebra 109 (1996), 143-195.

[20] A. Meurman and M. Primc, Annihilating fields of standard modules of $s l(2, \mathbb{C})^{-}$and combinatorial identities, Mem. Amer. Math. Soc. 137, AMS, Providence RI, 1999.

[21] O. Perše, Vertex operator algebras associated to type $B$ affine Lie algebras on admissible half-integer levels, J. Algebra 307 (2007), 215-248.

[22] O. Perše, Vertex operator algebras associated to certain admissible modules for affine Lie algebras of type A, Glas. Mat. Ser. III 43(63) (2008), 41-57.

[23] Y.-C. Zhu, Modular invariance of characters of vertex operator algebras, J. Amer. Math. Soc. 9 (1996), 237-302.

O. Perše

Department of Mathematics

University of Zagreb

10000 Zagreb

Croatia

E-mail: perse@math.hr

Received: 15.5.2012. 